\title{
Designing a Bi-objective Integrating Mathematical Model for Dynamic Sustainable Cellular Manufacturing Systems Considering Production Planning
}

\author{
Ghodsi $\mathbf{R}^{1 *}$, Mostafayi $\mathrm{S}^{2}$, Mansouri $\mathrm{Z}^{2}$ and Bakhtiari $\mathbf{M}^{2}$ \\ ${ }^{1}$ Engineering Department, Central Connecticut State University, USA \\ ${ }^{2}$ Department of Industrial Engineering, Kurdistan University, Sanandaj, Iran
}

\begin{abstract}
Increasing the profits of producers solely or alternatively minimizing production costs for manufacturers were considered in classical models for planning and designing cellular manufacturing systems. Provided that, with the expansion of manufacturing systems and the increasing demand for products, devastating effects of increasing levels of greenhouse gases on the environment has become more evident and has gained the attention of governments. Besides, the satisfaction of workers' working conditions, sound pollution and other issues, are vital to establish a sustainable manufacturing system. In this paper, a multi-objective mathematical model (linear/nonlinear) is presented to create stable cellular manufacturing system regarding to mentioned matters in order to minimize the costs of system establishment in addition to optimizing the environmental impacts of the manufacturing system. This model is introduced as a stable cellular manufacturing system (SCMS) and in fact is seeking for a solution to create a manufacturing system in which all factors such as producers, the environment, workers and other are beneficiaries. Also, Epsilon Constraint/LP metric/Pareto Front multi-objective approach given the weights of objectives is used to solve the model.
\end{abstract}

Keywords: Dynamic cellular manufacturing system; Sustainable system; Production planning; Mathematical programming; LP metric

\section{Introduction}

Manufacturing strategies and approaches have significantly changed in recent decades in comparison to the first half of the 20th century. One of the changes that has been employed and implemented by numerous companies in recent decades is the Group Technology (GT) and Cellular Manufacturing (CM) concepts. Group technology (GT) is a management theory that aims to group products with similar process or manufacturing characteristics, or both. Cellular manufacturing system (CMS) is a manufacturing concept where aims to group products to part families based on their similarities is manufacturing processing and also, machines are grouped to machine cells based on parts manufactured by them. CMS framework is a major application of group technology (GT) philosophy. The basic purpose of $\mathrm{CM}$ is to identify machine cells and part families concurrently, and to assign part families to machine cells in order to minimize the intercellular and intracellular costs of parts. Some real-world limitations in CF are: available capacity of machines must not be exceeded, safety and technological necessities must be met, the number of machines in a cell and the number of cells have not be exceeded an upper bound, intercellular and intracellular costs of handling material between machines must be minimized, machines must be utilized in effect [1].

Some researchers also suggest the benefits of implementing the cell production as: Reducing setup time, reduce parts inventory under manufacturing, reducing the cost of parts movements, increasing the efficiency of machinery. Flexible manufacturing system (FMS) is a highly integrated manufacturing system. The relation between its components is very complex. The mathematical programming approaches are very difficult to solve for very complex system so the simulation of FMS is widely used to analyze its performance measures. Also the FMS components are very sophisticated and costly. If FMS has to be implemented then it is better to analyze its results using simulation which involves no loss of money, resource and labor time. As a typical discrete event system FMS have been studied in such aspects as modeling and performance analysis.

Zeballos, Quiroga, and Henning (2010) proposed a constraint programming $(\mathrm{CP})$ formulation that simultaneously considers machine loading, part routing, tool allocation and operation scheduling in FMS environments. It employs two different two-index variables in order to model machining activities, instead of a four index one. This feature considerably reduces the dimensionality of the approach and facilitates the modeling of machine and tool specific constraints. Furthermore, the proposal represents tool management features in terms of tool types. Indeed, the tool instances demand is calculated indirectly, based on tool type, tool life-time, and tool magazine constraints [2]. Xing, Han, Zhou, and Wang (2012) embedded a deadlock avoidance policy into genetic algorithm to develop a deadlock-free scheduling algorithm for FMSs to minimize the makespan [3].

In classical models of planning and designing cell production systems and also flexible manufacturing, only increasing the manufacturing profit or alternatively minimizing production costs for manufacturers was considered and they only consider the manufacturer and the economy and social rights of workers and the environment are not significant. While with the expansion of production systems and the

*Corresponding author: Ghodsi R, Engineering Department, Central Connecticut State University, USA, Tel: +1 860-832-3200; E-mail: ghodsi@ccsu.edu

Received October 27, 2015; Accepted October 29, 2015; Published November 10,2015

Citation: Ghodsi R, Mostafayi S, Mansouri Z, Bakhtiari M (2015) Designing a Bi-objective Integrating Mathematical Model for Dynamic Sustainable Cellular Manufacturing Systems Considering Production Planning. J Appl Mech Eng 4: 184. doi:10.4172/2168-9873.1000184

Copyright: ( 2015 Ghodsi R, et al. This is an open-access article distributed under the terms of the Creative Commons Attribution License, which permits unrestricted use, distribution, and reproduction in any medium, provided the original author and source are credited. 
increasing demand for production economic and social impacts caused and also the devastating effects of increasing greenhouse gases on the environment was more evident and considered by the government. As the most basic definition of the supply chain:

Supply chain management (SCM) spans all movements and storage of raw materials, work-in-process inventory, and finished goods from the point-of-origin to the point-of-consumption [4]. In the past, supply chain approaches has been used only in order to determine the optimum location, facilities management, inventory management, the amount of current passing through the facility and etc. that only economic factors were considered. But over time and with the development of issues such as global warming, water and ground pollution, increasing greenhouse gases and etc. environmental protection and related strategies is considered as one of the most important factors in organizations and led to creation of a new concepts in the supply chain as Environmental Supply Chain management (ESCN) where in addition to taking into account the objectives of profit maximization or minimization $\mathrm{n}$ of the cost, environmental considerations are also included in the objective functions and constraints which leads to the integration of environmental factors with facilities, transportation methods, processes, product design, technology selection and etc. Later, with the expansion of the EU to support human rights, social issues were also considered in supply chain topics.

Today, a lot of research has been done to assess supply chain performance based on the three objectives of economic, environmental performance and social responsibility which has led to integration of the concept of sustainability with supply chain. Sustainability itself means: Sustainability is commonly defined as utilizing resources to meet the needs of the present without compromising future generations' ability to meet their own need [5]. 12 different definitions are offered for the sustainable supply chain until 2013 [6], including: Sustainable Supply Chain Management (SSCM) is defined as a set of managerial practices that include all of the following:

- Environmental impact as an imperative;

- Consideration of all stages across the entire value chain for each product;

- A multi-disciplinary perspective, encompassing the entire product life-cycle.

This definition implies a few broad themes in our perspective on environmental sustainability. First, firms must view environmental impact of their activities as an integral part of decision-making, rather than as a constraint imposed by government regulation or social pressure, or as a fad to exploit by appearing to be "green". Second, firms must pay attention to environmental impact across the entire value chain, including those of suppliers, distributors, partners and customers. Third, firms' view of sustainability must transcend a narrow functional perspective and encompass a broader view that integrates [7]. also, Sustainable SCM is the management of material, information and capital flows as well as cooperation among companies along the supply chain while integrating goals from all three dimensions of sustainable development, i.e., economic, environmental and social, which are derived from customer and stakeholder requirements. In sustainable supply chains, environmental and social criteria need to be fulfilled by the members to remain within the supply chain, while it is expected that competitiveness would be maintained through meeting customer needs and related economic criteria [8]. Now we want to provide the main concepts of the three main areas of sustainable supply chain.

\section{Economic dimension (economic sustainability)}

As only papers dealing with supply chain management are taken into account, it is logical that economic issues are addressed. Most often, total cost or net revenue is taken as indicators. Yet, there are a number of papers not providing insights into what kind of economic goals is pursued [9]. In fact, in some ways that is the traditional supply chain that includes economic concept such as the profits, loss, costs and etc.

\section{The environment (environmental sustainability)}

Green or environmentally sustainable supply chain management (ESCM) has been viewed as one area where organizations and industry can make significant contribution to both economic and environmental development [10]. ESCM has been defined as the explicit consideration of ecological dimensions in the planning, operations, and management of SCs [11]. Most environmental aspects that are combined with sustainability include: carbon emissions, energy consumption and waste generation. In many papers, life-cycle assessment data forms the starting point for the analysis. Hence, energy demand and $\mathrm{CO} 2-$ emissions e.g., [12-15] are among the frequently mentioned topics. Yet, in a number of cases, rather comprehensive lists of environmental impact criteria are taken up, such as referring to all kinds of natural capital e.g., [16] or resources, such as water or energy as well as waste [9,17-19].

\section{Social dimension (social sustainability)}

Social sustainability has been examined to a far lesser degree than environmental or green supply chain management [20]. Social sustainability in supply chains addresses issues of social justice and human rights with studies focusing on practices such as supplier human rights actions, labor conditions, codes of practices and social auditing, supplier compliance with child labor laws, and the delivery of social equity through sourcing from diverse suppliers in terms of gender, size, ethnicity and avoidance of conflicts of interest. Including social aspects in network design decisions allows to better evaluate the impact of a supply chain on its stakeholders: employees, customers and local communities. This also helps obtaining consistency between qualitative and quantitative decision [21]. According to the description mentioned sustainability issues should be considered and examined in production. Therefore we address this issue and the need to be considered.

\section{Problem Definition}

The concept of sustainability in the supply chain means meeting the economic needs and social and environmental sustainability which occurs with the flow of goods and services between suppliers, manufacturers and customers. Sustainability could be studied in economic, social and environmental areas. In the economic area, the main objective is cost minimization or profit maximization. With the pass of the time and paying attention to environmental issues decision-makers combined environmental factors with the decisionmaking process. These environmental factors could include various transportation equipment, production process, product design, production technology selection and etc. However, social sustainability has been studied less than two other areas, its impact cannot be overlooked. The workers social rights are studied in this area. In the current issue social sustainability concerns job security of workers which is applied by determining coefficients for hiring and firing workers. Adverse impact on the increasing level of unemployment of workers is considered using these coefficients and it is tried to minimize 
it. The proposed model is sustainable and dynamic cell production which considers two main objectives: the first objective is to minimize the production system costs including part manufacturing time costs, the costs of moving parts inside and outside cells, storage and lag costs of goods, the cost of workers' wages and the cost of purchasing machinery and the second objective is about minimizing the impact of sustainability criteria on environment. Because in the real world, each piece produced in the industry have a negative impact on the environment and society. In this paper for the production of any of the products different technologies has been considered and their selection depends on the production rate and also emission rate of environmental pollutants. Each technology has a special environmental factor to show the desired effect of technology on the environment and as for the use of any production technology some facilities such as machinery and etc. are required technology selection should be based on the amount of resources available. In this model, unlike the classic model of the cell production that the distance between cells are considered the same, these intervals are different and this leads the problem to approach the real world and it gets more flexible.

The considered problem takes into consideration three aspects of sustainability simultaneously. This means that in addition to considering the lowest cost, the least amount of environmental pollutants and the least adverse impact on labor satisfaction are considered. Coefficients intended to show the environmental impact technologies firing workers and the impact of each product on society, and according to different economic views among economists and experts, these coefficients are applied in order to establish a stable production system.

In the real world there are many examples to show that any product has a certain share in the economy of society. In fact, regardless of the profitability of certain goods, for reasons such as indigenousness and the obligation to keep the production industry of that product, some goods should be produced. Another major innovation in this study is paying attention to this issue that an economic factor is considered for each product to demonstrate the effectiveness of its production in community in a way that the impact factor of local products is a number greater than the imported products. In other words, each product has an effect coefficient on the economy. For optimum control of this effect considering manufactured goods, a term is added to the second objective function.

Generally, the problem is formulated by considering the following assumptions:

- The demand for each part in each period is determined.

- The capacity of each machine is determined.

- The overhead and maintenance cost for each machine is determined.

- System reconfiguration including adding or removing machinery to each cell and retransfer from one cell to another occurs during the period.

- The number of cells is determined and does not change in each period.

- Adverse impact of firing workers has been considered by applying corresponding economic factors.

- $\quad$ For each product, depending on the importance of a good rate of economic importance have been identified.

- Several technologies exist to produce each product depending on the amount of production and the amount of environmental pollution.

- An environmental factor is considered for each product.

- In each period a certain number of workers and machines are available.

\section{Notations:}
Subscripts:
$P \quad$ Number of part types.
W Number of worker types.
$M \quad$ Number of machine types.
$O_{p} \quad$ Number of operation types.
C Number of cells.
$H \quad$ Number of periods.
$T \quad$ Number of Technology.
$i \quad$ Index for part type $i$.
$J \quad$ Index for operation type $j$.
$w \quad$ Index for worker type $w$.
$m \quad$ Index for machine type $m$.
$C \quad$ Index for cell $c$.
$h \quad$ Index for period $h$.
$T$ Index for Technology $t$.

\section{Input parameters:}

$l_{j i m w}\left\{\begin{array}{l}1 \text { if machine type } \mathrm{m} \text { is able to process } j \text { th opertion of part type } i \text { with worker } w \\ 0 \text { other }\end{array}\right.$ $b_{i j}\left\{\begin{array}{l}1 \text { if part type } i \text { needs operation type } j \\ 0 \text { other }\end{array}\right.$ types.

$\min M_{c}$ Minimum size of cell $c$ in terms of the number of machine types.

$\max M_{c}$ Maximum size of cell $c$ in terms of the number of machine

$\min W_{c}$ Minimum size of cell $c$ in terms of the number of workers.

$A M_{m}$ Number of available machines type $m$.

$C H T_{t}$ Acquisition cost of handling technology $t$.

$U H T_{t}$ Utilization of handling technology $t$.

$T M_{m t}$ Number of Machine type $\mathrm{m}$ are used for technology $\mathrm{t}$.

$C M T_{t}$ Acquisition cost of manufacturing technology $t$.

$M E P T_{i}$ Manufacturing emissions of each unit part $i$.

$M E T_{t}$ Per unit environmental impacts of manufacturing technology $t$.

$H E T_{t}$ Per unit environmental impacts of handling technology $t$.

$E I P_{i}$ Per unit economic impacts of production of part type $i$.

$E I W_{w}$ Per unit economic impacts of firing of workers $w$. 
$A W_{w}$ Number of available workers type $w$.

$W T_{w h}$ Available time for worker type $w$ in period $h$.

$M T_{m h}$ Available time for machine type $m$ in period $h$.

$t_{i j m w}$ Processing time of $j$ th operation for part type $i$ on machine type $m$ with worker type $w$.

$D_{i h}$ Demand of part type $i$ in period $h$.

$\theta_{i t}^{\text {inter }}$ Unit material handling cost inter cells of each part type $i$ with handling technology $t$.

$\theta_{i}^{\text {intra }}$ Unit material handling cost intra cells of each part type $i$.

$\gamma_{i h}$ Unit holding cost of part type $i$ in period $h$.

$\lambda_{i h}$ Unit backorder cost of part type $i$ in period $h$.

$\delta_{m}^{\text {Ins }}$ Installing cost of machine type $m$.

$\eta_{m}^{\text {Ins }}$ Removing cost of machine type $m$.

$A D_{c, c^{\prime}}$ average distance between candid places belonging to cell $\mathrm{c}$ if $\left(c=c^{\prime}\right)$

$E C_{c, c^{\prime}} 1$ if $\left(c \neq c^{\prime}\right)$ and 0 if $\left(c=c^{\prime}\right)$

$\alpha_{m}$ Maintenance and overhead costs of machine type $m$.

$\beta_{m}$ variable cost for each work time unit for machine type $m$

$S_{w h}$ Salary cost of worker type $w$ in period $h$.

$H I_{w h}$ Hiring cost of worker type $w$ within period $h$.

$F_{w h}$ Firing cost of worker type $w$ in period $h$.

A large positive number

\section{Decision variable}

$X_{j i m w c h}\left\{\begin{array}{l}1 \text { if } j \text { th } \text { operation of part type } i \text { is performed by the on machine type } m \text { with worker } w \text { in cell } c \text { in period } h \\ 0 \text { other }\end{array}\right.$

$\operatorname{MaT}_{t}\left\{\begin{array}{l}1 \text { if manufacturing technology } t \text { tobe used } \\ 0 \text { other }\end{array}\right.$

$\operatorname{HaT}_{t}\left\{\begin{array}{l}1 \text { if handling technology } t \text { tobe used } \\ 0 \text { other }\end{array}\right.$

$N M_{m c h}$ Number of machines type $m$ allocated to cell $c$ in period $h$.

$N W_{w c h}$ Number of workers type $w$ allocated to cell $c$ in period $h$.

$P_{i h}$ Production volume of part type $i$ to be produced in period $h$.

$I_{i h}$ Inventory of part type $i$ at the end of period $h ; I_{i 0}=0$.

$B_{i h}$ Backorder of part type $i$ in period h; $B_{i 0}=0$.

$K_{m c h}^{+}$Number of machines type $m$ added to cell $c$ during period $h$.

$K_{m c h}^{-}$Number of machines type $m$ removed from cell $c$ during period $h$.

$L_{w c h}^{+}$Number of workers type $w$ added to cell $c$ during period $h$.

$L_{w c h}^{-}$Number of workers type $w$ removed from cell $c$ during period $h$.

$\operatorname{Min} Z=Z_{1}+Z_{2}+Z_{3}+Z_{4}+Z_{5}+Z_{6}+Z_{7}+Z_{8}+Z_{9}+Z_{10}+Z_{11}+Z_{12}+Z_{13}$

$Z_{1}=\sum_{i=1}^{Q} \sum_{m=1}^{M} \sum_{w=1}^{W} \sum_{c=1}^{C} \sum_{h=1}^{H} \beta_{m} t_{i j m w} P_{i h} X_{j i m w c h}$
$Z_{2}=\sum_{t=1}^{T} \sum_{i=1}^{Q} \sum_{j=1}^{O p-1} \sum_{w=1}^{W} \sum_{c=1}^{C} \sum_{c^{\prime}=1}^{C} \sum_{h=1}^{H} \theta_{i t}^{i n t r a} H a T_{t} P_{i h} A D_{c c^{\prime}} E C_{c, c^{\prime}}\left(\sum_{m=1}^{M} X_{j i m w c h}\right)\left(\sum_{m=1}^{M} X_{(j+1) i m w c^{\prime} h}\right)$

$Z_{3}=\sum_{i=1}^{Q} \sum_{j=1}^{O p-1} \sum_{w=1}^{W} \sum_{c=1}^{C} \sum_{c^{\prime}=1}^{C} \sum_{h=1}^{H} \theta_{i}^{\text {inter }} P_{i h} A D_{c c^{\prime}}\left(1-E C_{c, c^{\prime}}\right)\left(\sum_{m=1}^{M} X_{j i m w c h}\right)\left(\sum_{m=1}^{M} X_{(j+1) i m w c^{\prime} h}\right)$

$Z_{4}=\sum_{h=1}^{H} \sum_{i=1}^{Q} \vartheta_{i h} I_{i h}$

$Z_{5}=\sum_{h=1}^{H} \sum_{i=1}^{Q} \lambda_{i h} B_{i h}$

$Z_{6}=\sum_{h=1}^{H} \sum_{c=1}^{C} \sum_{m=1}^{M} \alpha_{m} N M_{m c h}$

$Z_{7}=\sum_{h=1}^{H} \sum_{c=1}^{C} \sum_{m=1}^{M} \delta_{m}^{I n s} K_{m c h}^{+}$

$Z_{8}=\sum_{h=1}^{H} \sum_{c=1}^{C} \sum_{m=1}^{M} \eta_{m}^{\text {Ins }} K_{m c h}^{-}$

$Z_{9}=\sum_{h=1}^{H} \sum_{c=1}^{C} \sum_{w=1}^{W} S_{w h} N W_{w c h}$

$Z_{10}=\sum_{h=1}^{H} \sum_{c=1}^{C} \sum_{w=1}^{W} H I_{w h} L_{w c h}^{+}$

$Z_{11}=\sum_{h=1}^{H} \sum_{c=1}^{C} \sum_{w=1}^{W} F_{w h} L_{w c h}^{-}$

$Z_{12}=\sum_{t=1}^{T} C M T_{t} M a T_{t}$

$Z_{13}=\sum_{t=1}^{T} \frac{C H T_{t}}{U H T_{t}} H a T_{t}$

$\operatorname{Min} W=W_{1}+W_{2}+W_{3}+W_{4}+W_{5}$

$W_{1}=\sum_{i=1}^{I} \sum_{h=1}^{H} P_{i h} M E P T_{i}$

$W_{2}=\sum_{t=1}^{T} M E T_{t} M a T_{t}$

$W_{3}=\sum_{t=1}^{T} H E T_{t} H a T_{t}$

$W_{4}=\sum_{i=1}^{I} \sum_{h=1}^{H} P_{i h} \frac{1}{E I P_{i}}$

$W_{5}=\sum_{w=1}^{W} \sum_{c=1}^{C} \sum_{h=1}^{H} L_{w c h}^{-} E I W_{w}$ 


$$
\begin{aligned}
& \sum_{i=1}^{Q} \sum_{m=1}^{M} \sum_{j=1}^{O p} X_{j i m w c h} t_{i j m w} P_{i h} \leq N W_{w c h} W T_{w h} \quad \forall w, c, h \\
& \sum_{i=1}^{Q} \sum_{w=1}^{M} \sum_{j=1}^{O p} X_{i m w c h} t_{i j m w} P_{i h} \leq N M_{m c h} M T_{m h} \quad \forall m, c, h \\
& D_{i h}=P_{i h}+I_{i, h-1}-B_{i, h-1}-I_{i h}+B_{i h} \quad \forall i, h \\
& \sum_{c=1}^{C} X_{j i m w c h} \leq l_{j i m w} \quad \forall i, m, w, h \\
& \sum_{c=1}^{C} \sum_{w=1}^{W} \sum_{j=1}^{O p} X_{j i m w c h}=b_{j i} \quad \forall i, j, h \\
& \sum_{c=1}^{C} \sum_{m=1}^{M} \sum_{w=1}^{W} \sum_{j=1}^{O p} X_{j i m w c h} \leq A . P_{i h} \quad \forall i, h \\
& N M_{m c, h-1}+K_{m c h}^{+}-K_{m c h}^{-}=N M_{m c h} \quad \forall m, c, h \\
& \sum_{c=1}^{C} N M_{m c h} \leq A M_{m} \quad \forall m, h \\
& \sum_{c=1}^{C} N M_{m c h} \geq \min M_{m} \quad \forall m, h \\
& \sum_{m=1}^{M} N M_{m c h} \leq \max _{c} \quad \forall c, h \\
& N W_{w c, h-1}+L_{w c h}^{+}-L_{w c h}^{-}=N W_{w c h} \quad \forall w, c, h \\
& \sum_{c=1}^{C} N W_{w c h} \leq A W_{c} \quad \forall w, c, h \\
& \sum_{w=1}^{W} N W_{m c h} \geq \min W_{c} \quad \forall m, c, h \\
& \sum_{i=1}^{Q} \sum_{j=1}^{O p} \sum_{w=1}^{W} \sum_{c=1}^{C} \sum_{h=1}^{H} X_{j i m w c h} \leq A . \text { Tech }_{t} T M_{m t} \\
& \sum_{t=1}^{T} H a T_{t}=1 \\
& \sum_{t=1}^{T} M a T_{t}=1 \\
& X_{\text {jimwch }}, \operatorname{MaT}_{t}, \operatorname{HaT}_{t} \in\{0,1\} \\
& \forall j, i, m, w, c, h \\
& N M_{m c h}, K_{m c h}^{+}, K_{m c h}^{-} \geq 0 \text { and integer } \quad \forall m, c, h \\
& N W_{w c h}, L_{w c h}^{+}, L_{w c h}^{-} \geq 0 \text { and integer } \quad \forall w, c, h \\
& P_{i h}, B_{i h}, I_{i h} \geq 0 \text { and integer } \quad \forall i, h
\end{aligned}
$$

The proposed model has two objective functions that the first one includes thirteen terms and expresses the system costs. The first term describes the cost of production time of parts. The second and third terms calculate the cost of parts movements inside and outside the cells. The fourth and fifth terms explain the storage and lag costs of goods respectively. The costs related to the purchase of machinery are shown by the sixth term of the objective function. Since the model is formulated as a multi-period one, therefore it is possible to change the machinery in order to increase the flexibility of the system over different periods. Costs imposed by this issue are stated by the seventh and eighth term of the objective function. The cost of labor salary is calculated by the ninth term. During different periods labor transfer or actually hiring and firing is done in order to reduce transport costs. The costs related to this policy are stated in tenth and eleventh terms.

The second objective function includes five terms that focuses on meeting model sustainability criteria. Because in the real world, each parts produced in industry has an impact on the environment and society, in particular, it is desirable for this impact to be minimum. The first term of the second objective function deals with minimization of this impact coefficient. Also, each part can be produced using different technologies. Naturally, each technology has effects such as environmental pollution, economic effects, etc. These effects are controlled by the second term of the objective function. Similarly, various technologies are available to transport the goods that each one effects similar to production technology. The third term of the objective function controls these effects. There are different examples in the real world that shows any good that are produced, has a certain proportion in economics of the society. In fact, regardless of the profitability some goods must be produced for reasons such as indigenous and the obligation to keep the product. In the other words each good has an impact coefficient in economy. The fourth term of the second objective function is utilized in order to optimal control of this impact. Similarly an impact could be considered for hiring and firing workers also affecting society and economy. In other words, workers firing affects the economy and the unemployment rate that is controlled using the last term of the second objective function.

Constraints (1) and (2) ensure that the available time for workers and capacities of machines are not exceeded, respectively. Balance in the amount of part type $i$ between two consecutive periods is shown in constraint (3). It is clear that if $\mathrm{I}_{\mathrm{ih}}>0$, a holding cost is incurred and if $\mathrm{B}_{\mathrm{ih}}>0$, a backorder cost is incurred. Constraints (4) and (5) imply that only one worker is allotted for processing each part on each machine type. This model is flexible to enable a worker to work on several machines. This means that, if one part is required to one machine type to be processed, more than one worker will be able to service this machine type. Constraint (6) ensures that no cell, machine, and worker should be considered if production volume of part $i$ in period $h$ equals to 0 . Constraint (7) guarantees that the number of machines in the current period is equal to the number of machines in the previous period plus the number of machines being moved in, minus the number of machines being moved out. Constraint (8) guarantees that the total number of machines of each type allocated to different cells in each period will not exceed the total number of available machines of that type. The lower and upper bounds for number of machines which are assigned to each cell in each period are specified in constraint (9) and (10). Similar to constraint (7), constraint (11) balances the number of each worker type in each period and cell. Similar to constraint (8, constraint (12) states the limitation in number of each worker type in each period and cell. Constraint (13) ensures that the number of workers assigned to cell $\mathrm{c}$ in each period is at least $\min W_{c}$.

Since some facilities such as machinery, etc. are required to use any technology, technology selection should be based on the amount of resources available. The constraint (14) limits the selection of each technology to the resources available. Constraints (15) and (16) states 
that in order to produce the goods simultaneously only one production and transportation technology could be used. Finally the type and classification of utilized variables are expressed in constraints (17) to (20).

Linearization: The proposed model is nonlinear due to the terms (1), (2), and (3) in the objective function (I) and constraints (1) and (2). New class of variables added to the model is defined as follows:

$$
\begin{aligned}
& X_{\text {jimwch }} P_{i h}=X P_{j i m w c h} \\
& \left(\sum_{m=1}^{M} X_{j i m w c h}\right)\left(\sum_{m=1}^{M} X_{(j+1) i m w c^{\prime} h}\right) P_{i h}=J_{j i w c c^{\prime} h} \\
& J_{j i w c c^{\prime} h} H a T_{t}=J H_{j i w c c^{\prime} h}
\end{aligned}
$$

New constraints added to the model are as follows:

$$
\begin{aligned}
& X P_{j i m w c h} \geq P_{i h}-A\left(1-X_{j i m w c h}\right) \\
& X P_{j i m w c h} \leq P_{i h}+A\left(1-X_{j i m w c h}\right) \\
& J_{j i w c c^{\prime} h} \geq P_{i h}-A\left(2-\left(\sum_{m=1}^{M} X_{j i m w c h}\right)-\left(\sum_{m=1}^{M} X_{(j+1) i m w c^{\prime} h}\right)\right) \\
& J_{j i w c c^{\prime} h} \leq P_{i h}+A\left(2-\left(\sum_{m=1}^{M} X_{j i m w c h}\right)-\left(\sum_{m=1}^{M} X_{(j+1) i m w c^{\prime} h}\right)\right) \\
& J H_{j i w c c^{\prime} h} \geq J_{j i w c h}-A\left(1-H a T_{t}\right) \\
& J H_{j i w c c^{\prime} h} \leq J_{j i w c h}+A\left(1-H a T_{t}\right) \\
& X P_{j i m w c h}, J_{j i w c c^{\prime} h}, J H_{j i w c c^{\prime} h} \geq 0 \text { and is integer }
\end{aligned}
$$

The linearized programming model is as follows:

$$
\begin{aligned}
& \text { Min }=\sum_{i=1}^{13} Z_{i} \\
& Z_{1}=\sum_{i=1}^{Q} \sum_{m=1}^{M} \sum_{w=1}^{W} \sum_{c=1}^{C} \sum_{h=1}^{H} \beta_{m} t_{i j m w} X P_{j i m w c h} \\
& Z_{2}=\sum_{t=1}^{T} \sum_{i=1}^{Q} \sum_{j=1}^{O p-1} \sum_{w=1}^{W} \sum_{c=1}^{C} \sum_{c^{\prime}=1}^{C} \sum_{h=1}^{H} \theta_{i t}^{\text {intra }} A D_{c c^{\prime}} E C_{c, c^{\prime}} J H_{j i w c c^{\prime} h} \\
& Z_{3}=\sum_{i=1}^{Q} \sum_{j=1}^{O p-1} \sum_{w=1}^{W} \sum_{c=1}^{C} \sum_{c^{\prime}}^{C} \sum_{h=1}^{C} \theta_{i}^{H} \theta^{\text {inter }} A D_{c c^{\prime}}\left(1-E C_{c, c^{\prime}}\right) J_{j i w c c^{\prime} h} \\
& \text { Min }=\sum_{i=1}^{5} W_{i} \\
& \text { s.t }
\end{aligned}
$$$$
\text { (3) - (20) }
$$

Constraint 1:$$
\sum_{i=1}^{Q} \sum_{m=1}^{M} \sum_{j=1}^{O p} X P_{j i m w c h} t_{i j m w} \leq N W_{w c h} W T_{w h}
$$

Constraint 2:

$$
\sum_{i=1}^{Q} \sum_{w=1}^{M} \sum_{j=1}^{O p} X P_{i m w c h} t_{i j m w} \leq N M_{m c h} M T_{m h}
$$

The proposed process to solve the model: The model presented in the previous section is a multi-objective planning problem. First we should change the problem to an equivalent problem with one objective function. Here LP-metric method, a common approach used to solve multi-objective models, the problem could be replaced by one objective function. In this way the distance between objectives and their corresponding optimal objectives are minimized.

$$
\begin{aligned}
& \operatorname{Min} Z(x)=\left[Z_{1}(x), Z_{2}(x), \ldots, Z_{p}(x)\right] \\
& \text { s.t } x \in X
\end{aligned}
$$

In general, the objective function is defined as the following:

$$
\operatorname{Min} L_{p}=\left\{\sum_{j=1}^{K} \alpha_{j}\left[z_{i}-z_{i}^{*}\right]^{p}\right\}^{1 / p}
$$

Where if $\alpha_{i}=1 \forall i$, the weight of the objective function is assumed to be equal.

In this problem, because the first two objectives are not scaled the same as each other, they are normalized using the following equation where $Z_{i}^{*}$ is the optimum value of the objective function. Both objective functions are replaced by the following equation for the optimum model proposed and leads the problem to have one objective function. In this study, we assume that the objective functions are named as $\mathrm{Z}_{1}, \mathrm{Z}_{2}$. Based on LP-metric method, sustainable optimization model of dynamic cell production problem is solved for each of these two objective functions separately. The objective function of LP-metric model can be formulated as follows:

$$
\operatorname{Min} Z_{3}=\left[\alpha \frac{z_{1}-z_{1}^{*}}{z_{1}^{*}}\right]+\left[(1+\alpha) \frac{z_{2}-z_{2}^{*}}{z_{2}^{*}}\right]
$$

Where $0 \leq a \leq 1$. Weights coefficients are related to objective function elements mentioned above. Using the above equation, the problem has one objective function which can be easily solved. Because of the importance of the two objective functions, total costs and total man-hour distortions between the cells simultaneously, three models are presented for sensitivity analysis as the following:

1. $Z_{1}$ model: includes total costs of design problem and cell production formation with corresponding coefficients.

2. $\mathrm{Z}_{2}$ model: includes factors related to production systems sustainability.

$\mathrm{Z}_{3}$ model: $L-P$ metricmodel, is a combination of $\mathrm{Z}_{1}$ and $\mathrm{Z}_{2}$ model with corresponding constraints.

So a series of multi-objective solutions are obtained for the different values of $\alpha$. In order to gain the optimal Pareto front, these solutions can be calculated for different amounts of $L-P$ metric problem.

\section{Conclusions and Suggestions for Future Research}

Due to the increasing and inevitable demand for production of different goods in human societies and manufacturers effort to produce qualifies products in order to increase profitability and also the importance of the emissions of greenhouse gasses caused by production systems the need to plan to create a balance between production and the environment is considered more than ever. In addition, in order to have a stable system that all levels of society are the beneficiaries, other aspects of workers' satisfaction with working conditions, the economic impact of each product, the labor hiring and firing effect and other items should be considered specially. In this paper, a mathematical 
Citation: Ghodsi R, Mostafayi S, Mansouri Z, Bakhtiari M (2015) Designing a Bi-objective Integrating Mathematical Model for Dynamic Sustainable Cellular Manufacturing Systems Considering Production Planning. J Appl Mech Eng 4: 184. doi:10.4172/2168-9873.1000184

model considering all the factors listed has been proposed as sustainable cell production model leads to job stability of workers with the least amount of hiring and firing and reduce environmental pollutants emissions. On the other hand, this model focuses on production of some goods affecting the regional economy and somehow their import must be banned. Since this issue is introduced for the first time in this paper, for future research we can solve this problem under conditions of uncertainty. Also it is suggested to use the features available in proposed model in order to study the production systems in the real world as case studies. Given the probable complexity of the problem innovative and meta-heuristic algorithms can be used to develop the right solutions and compare the results.

\section{References}

1. Heragu S (1997) Facilities design. Boston MA: PWS Publishing Company 316

2. Zeballos LJ, Quiroga OD, Henning GP (2010) A constraint programming mode for the scheduling of flexible manufacturing systems with machine and tool limitations. Engineering Applications of Artificial Intelligence 23: 229-248.

3. Xing KY, Han LB, Zhou MC, Wang F (2012) Deadlock-free genetic scheduling for automated manufacturing systems based on deadlock control policy. IEEE Transaction on System Man, and Cybernetics Part B: Cybernetics 42: 603-615.

4. Simchi-Levi D, Kaminsky P, Simchi Levi E, Simchi-Levi E (2004) Managing the supply chain: The definitive guide for the business professional. New York: McGraw-Hill.

5. (1987) WCED - World Commission on Environment and Development, Our Common Future. Oxford Univ Press Oxford.

6. Ahi P, Searcy C (2013) A comparative literature analysis of definitions for green and sustainable supply chain management. Journal of Cleaner Production 52: 329-341.

7. Gupta S, Palsule-Desai OD (2011) Sustainable supply chain management: Review and research opportunities. IIMB Management Review 23: 234-245.

8. Mentzer JT, DeWitt W, Keebler JS, Min S, Nix NW, et. al. (2001) Defining supply chain management. Journal of Business Logistics 22: 1-26.

9. Seuring $S$ (2013) A review of modeling approaches for sustainable supply chain management. Decision Support Systems 54: 1513-1520.
10. Varsei et al., 2014

11. Zhu Q, Sarkis J (2004) Relationships between operational practices and performance among early adopters of green supply chain management practice in Chinese manufacturing enterprises. Jounal of Operations Management 22 : 265-289.

12. Cholette S, Venkat K (2009) The energy and carbon intensity of wine distribution: A study of logistical options for delivering wine to consumers. Journal of Cleaner Production 17: 1-13.

13. Edwards JB, McKinnon AC, Cullinane SL (2010) Comparative analysis of the carbon footprints of conventional and online retailing: a "last mile" perspective. International Journal of Physical Distribution and Logistics Management 40: 103-123.

14. Tan $\mathrm{RBH}$, Khoo HH (2005) An LCA study of a primary aluminum supply chain Journal of Cleaner Production 13: 607- 618.

15. Neto JF, Bloemhof-Ruwaard JM, van Nunen JAEE, van Heck E (2008) Designing and evaluating sustainable logistics networks. International Journal of Production Economics 111: 195-208.

16. Ukidwe NU, Bakshi BR (2005) Flow of natural versus economic capital in industrial supply networks and its implications to sustainability. Environmental Science and Technology 39: 9759-9769.

17. Georgiadis P, Besiou M (2009) Environmental strategies for electrical and electronic equipment supply chains: which to choose? Sustainability 1: 722733

18. Louis YYL, Wu CH, Kuo TC (2007) Environmental principles applicable to green supplier evaluation by using multi-objective decision analysis. International Journal of Production Research 45: 4317-4331.

19. Kainuma Y, Tawara N (2006) A multiple attribute utility theory approach to lean and green supply chain management. International Journal of Production Economics 101: 99-108.

20. Seuring S, Müller M (2008) From a literature review to a conceptual frame work for sustainable supply chain management. Journal of Cleaner Production 16: 1699-710.

21. Eskandarpour M, Dejax P, Miemczyk J, Peton O (2015) Sustainable supply chain network design: An optimization oriented review. Omega 54: 11-32. 